\title{
Characterization of antimicrobial and hemolytic properties of short synthetic cationic lipopeptides based on QSAR/QSTR approach
}

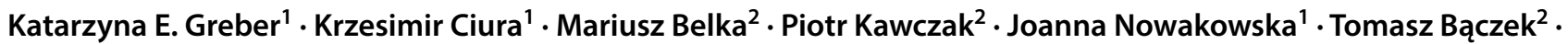 \\ Wiesław Sawicki ${ }^{1}$
}

Received: 23 October 2017 / Accepted: 12 December 2017 / Published online: 20 December 2017

(c) The Author(s) 2017. This article is an open access publication

\begin{abstract}
In this study, we investigated the influence of molecular descriptors of cationic lipopeptides on their antimicrobial activity and hemolytic properties. The quantitative structure-activity relationship and quantitative structure-property relationship models were constructed. The antimicrobial, hemolytic and retention data were used as dependent variable and structural parameters as the independent ones. The obtained results suggest that the chromatographic indexes can be employed for prediction of antibacterial activity and that lipopeptides present nonspecific interaction between erythrocytes and bacterial membranes.
\end{abstract}

Keywords QSAR · QSRR · Antimicrobial lipopeptides · MIC · Hemolysis

\section{Introduction}

Development of new antimicrobial agents is of the most important challenge these days. Antimicrobial peptides and lipopeptides (AMPs), which reveal serious therapeutic potential due to the broad spectrum of activity, rapid bacterial killing, and synergy with classical antibiotics, are seen to be very promising candidates. The antibacterial mode of action of peptides and lipopeptides is associated mostly with

Handling Editor: M. S. Palma.

Katarzyna E. Greber and Krzesimir Ciura contributed equally to this work.

Electronic supplementary material The online version of this article (https://doi.org/10.1007/s00726-017-2530-2) contains supplementary material, which is available to authorized users.

Katarzyna E. Greber

greber@gumed.edu.pl

1 Department of Physical Chemistry, Faculty of Pharmacy, Medical University of Gdańsk, Al. Gen. J. Hallera 107, 80-416 Gdańsk, Poland

2 Department of Pharmaceutical Chemistry, Faculty of Pharmacy, Medical University of Gdańsk, Al. Gen. J. Hallera 107, 80-416 Gdańsk, Poland the interactions with bacterial bilayer (Colomb-Cotinat et al. 2016; Greber and Dawgul 2017).

Lipophilicity of compounds is well known as a vital parameter in a quantitative structure-property relationship (QSPR), quantitative structure-activity relationship (QSAR) studies and quantitative structure-toxicity relationship (QSTR). A particular example of QSPR is a quantitative structure-retention relationship (QSRR) where the properties are defined as chromatographic parameters. The QSRR/ QSAR approach was successfully applied to predict antimicrobial activities of others class of antibiotics (Ciura et al. 2016).

The main aim of this study was to investigate how molecular descriptors influence the antimicrobial activity and hemolytic properties of short cationic lipopeptides. Additionally, QSRR models were built to evaluate the most important descriptors that influence the chromatographically determined lipophilicity of this class of chemicals.

\section{Experimental}

\section{Synthesis and purification}

Lipopeptides were synthesized, purified and analyzed according to the procedures described in details elsewhere (Greber et al. 2017). 


\section{MIC and MHC}

The minimum inhibitory concentration (MIC) was determined according to the procedure recommended by the [Clinical Laboratory Standards Institute (CLSI) (2012, 2017)]. The following Gram-positive strains were used: Staphylococcus aureus (ATCC 25923), S. epidermidis (PCM 2118), Bacillus subtilis (ATCC 6633), and Enterococcus faecalis (ATCC 29212). Minimum hemolytic concentration (MHC) was taken as the lowest concentration of lipopeptides which induced $10 \%$ of hemolysis of human red blood cells. Antimicrobial activity (MIC) toward Gram-positive strains and toxicity toward human red blood cells (MHC) are presented in supplementary materials in Table $1 \mathrm{~S}$.

\section{Chromatographic analysis}

The RP-HPLC experiments were performed on Shimadzu Prominence apparatus on a Chromolith ${ }^{\circledR}$ Performance RP-18 endcapped 100-4.6 mm monolithic column with a linear gradient 2-98\% phase B (where phase A was $0.1 \%$ TFA in water and phase $\mathrm{B}$ was $0.1 \%$ TFA in $\mathrm{ACN}$ ), at a flow rate of $2 \mathrm{~mL} / \mathrm{min}$, and UV detection at $214 \mathrm{~nm}$. The concentrations of lipopeptide samples were $100 \mu \mathrm{g} / \mathrm{mL}$ and the injected volume was $10 \mu \mathrm{L}$ (Greber et al. 2017).

\section{Molecular modeling}

HyperChem 8.08 (Hypercube, Waterloo, Canada) software was used for the calculation of molecular descriptors. The preliminary optimization of investigated compounds was carried out using the molecular mechanic calculations $(\mathrm{MM}+)$. In the next step, semi-empirical calculation method Austin Model 1 (AM1) was applied (HyperChem Computational Chemistry 1996). After calculation of molecular structures, Dragon 7.0 (Talete, Milan, Italy) software was used to calculate further set of constitutional indices, ring descriptors, the functional group counts, atom-centered fragments, atom-type E-state indices, CATS 2D, 2D Atom Pairs, molecular properties and charge descriptors (Dragon 7 molecular descriptors 2017; odeschini and onsonni 2009). Finally, 162 descriptors were used for analysis.

\section{QSAR/QSTR/QSRR analysis}

For the construction of QSAR, QSRR and QSTR models, multiple linear regression (MLR), partial least squares (PLS) and orthogonal partial least squares (OPLS) were applied (Roy et al. 2015; Worley and Powers 2013; Saxena and Prathipati 2003). During calculation, the log MIC value, $\log$ MHC and retention data $\left(\log _{k}\right)$ were used as dependent variable and structural parameters as the independent ones. In case of MLR calculation stepwise regression mode was chosen. This calculation was performed on Statistica software (Statistica 12, Statsoft, USA). The coefficient of correlation $(r)$ and determination $\left(R^{2}\right), F$ test value, standard deviation and the standard estimation error were used as the bases for testing the established MLR model. Next, using Simca software (Simca 13, Umetrix, Umea, Sweden) PLS and OLPS models were constructed (User Guide to SIMCA 2017). The validation of the established models was performed with leave-one out procedure based on $Q^{2}$ value (Alexander et al. 2015).

\section{Results and discussion}

Although the antimicrobial peptides are concerned as potential drugs, their mechanism of action is still not fully known. Several models have been proposed for last decades, including pore formation (Brogden 2005), detergent-like permeabilization of the bilayer (Bechinger and Lohner 2006), and membrane destabilization after AMPs coat the bilayer surface (Shai and Oren 2001). Judging the proposed models, it seems likely that there is no single mechanism which can explain AMP mechanism of action. Probably, AMPs of different chemical origins may be described by one or more of the above models (Horn et al. 2012). For this reason, the identification of the most important physicochemical descriptors, which affect the antimicrobial activities, is useful to gather the knowledge how the investigated class of AMPs works.

The dataset that includes calculated descriptors and chromatographic parameter $\log _{k}$ was used for QSAR analysis. Three regression methods MLR, PLS and OPLS were tested. Although both PLS and OPLS can be used for analysis of highly collinear data, the advantage of OPLS method in compression of PLS is an integrated orthogonal signal correction filter. The best QSAR models are obtained after OPLS calculation. The fifteen most important descriptors are listed in Table 1. All obtained models meet the Tropsha et al. (2003) criteria $\left(R^{2}>0.6\right.$ and $\left.Q^{2}>0.5\right)$. It is worth to notice that all obtained models are based practically on the same descriptors. This finding suggested that the mechanism of action against Gram-positive bacteria is nonspecific. The differences of MIC values obtained for each type of bacteria can be explained by the different affinity of bacterial membranes. The composition of lipid bilayer could be the main factor, which determines the higher activity of lipopeptides toward Staphylococcus epidermidis, and Bacillus subtilis than toward Staphylococcus aureus. The investigated lipopeptides showed the lowest activity against Enterococcus faecalis. In Table $2 \mathrm{~S}$, the lipidome map of tested strains is presented. The concentration of phosphatidylglycerols (PG), 
Table 1 List of molecular descriptors characterized by the highest VIP values in OPLS models built for QSAR models and QSTR model

\begin{tabular}{|c|c|c|c|}
\hline Descriptor & $\begin{array}{l}R^{2}=0.949 \\
\text { VIP }\end{array}$ & \multicolumn{2}{|l|}{$Q^{2}=0.890$} \\
\hline \multicolumn{4}{|l|}{ Bacillus subtilis } \\
\hline CATS2D_03_LL & 2.91 & $\begin{array}{l}\text { CATS (chemically advanced template search) 2D Lipophilic-Lipo- } \\
\text { philic at lag } 03\end{array}$ & CATS 2D \\
\hline CATS2D_04_LL & 2.91 & CATS2D Lipophilic-Lipophilic at lag 04 & CATS 2D \\
\hline H-046 & 2.83 & $\mathrm{H}$ attached to $\mathrm{C} 0(\mathrm{sp} 3)$ no $\times$ attached to next $\mathrm{C}$ & Atom-centered fragments \\
\hline CATS2D_02_LL & 2.83 & CATS2D Lipophilic-Lipophilic at lag 02 & CATS 2D \\
\hline CATS2D_05_LL & 2.81 & CATS2D Lipophilic-Lipophilic at lag 05 & CATS 2D \\
\hline $\mathrm{SssCH} 2$ & 2.79 & Sum of ssCH2 E-states & Atom-type E-state indices \\
\hline $\mathrm{SsCH} 3$ & 2.75 & Sum of ssCH3 E-states & Atom-type E-state indices \\
\hline ALOGP & 2.66 & Ghose-Crippen octanol-water partition coeff. $(\log P)$ & Molecular properties \\
\hline ALOGP2 & 2.53 & Squared Ghose-Crippen octanol-water partition coeff. (log $\left.P^{\wedge} 2\right)$ & Molecular properties \\
\hline CATS2D_01_LL & 2.52 & CATS2D Lipophilic-Lipophilic at lag 01 & CATS 2D \\
\hline CATS2D_06_LL & 2.44 & CATS2D Lipophilic-Lipophilic at lag 06 & CATS 2D \\
\hline $\log _{k}$ & 2.38 & HPLC retention factor & Experimental \\
\hline C-002 & 2.17 & $\mathrm{CH} 2 \mathrm{R} 2$ & Atom-centred fragments \\
\hline CATS2D_00_LL & 2.17 & CATS2D Lipophilic-Lipophilic at lag 00 & CATS 2D \\
\hline CATS2D_07_LL & 1.95 & CATS2D Lipophilic-Lipophilic at lag 07 & CATS 2D \\
\hline \multirow[t]{2}{*}{ Descriptor } & $R^{2}=0.949$ & $Q^{2}=0.860$ & \\
\hline & VIP & Full name & Block \\
\hline \multicolumn{4}{|c|}{ Enterococcus faecalis } \\
\hline CATS2D_03_LL & 3.11 & CATS2D Lipophilic-Lipophilic at lag 03 & CATS 2D \\
\hline CATS2D_04_LL & 3.11 & CATS2D Lipophilic-Lipophilic at lag 04 & CATS 2D \\
\hline ALOGP2 & 2.95 & Squared Ghose-Crippen octanol-water partition coeff. $\left(\log P^{\wedge} 2\right)$ & Molecular properties \\
\hline ALOGP & 2.95 & Ghose-Crippen octanol-water partition coeff. $(\log P)$ & Molecular properties \\
\hline H-046 & 2.85 & $\mathrm{H}$ attached to $\mathrm{C} 0(\mathrm{sp} 3)$ no $\times$ attached to next & Atom-centred fragments \\
\hline CATS2D_02_LL & 2.85 & CATS2D Lipophilic-Lipophilic at lag 02 & CATS 2D \\
\hline $\mathrm{SsCH} 3$ & 2.84 & Sum of ssCH3 E-states & Atom-type E-state indices \\
\hline CATS2D_05_LL & 2.84 & CATS2D Lipophilic-Lipophilic at lag 05 & CATS 2D \\
\hline $\mathrm{SssCH} 2$ & 2.78 & Sum of ssCH2 E-states & Atom-type E-state indices \\
\hline $\log _{k}$ & 2.76 & HPLC retention factor & \\
\hline CATS2D_01_LL & 2.39 & CATS2D Lipophilic-Lipophilic at lag 01 & CATS 2D \\
\hline CATS2D_06_LL & 2.33 & CATS2D Lipophilic-Lipophilic at lag 06 & CATS 2D \\
\hline BLTD48 & 2.18 & Verhaar Daphnia base-line toxicity from MLOGP (mmol/L) & Molecular properties \\
\hline BLTF96 & 2.18 & Verhaar Fish base-line toxicity from MLOGP (mmol/L) & Molecular properties \\
\hline MLOGP & 2.18 & Moriguchi octanol-water partition coeff. $(\log P)$ & Molecular properties \\
\hline \multirow[t]{2}{*}{ Descriptor } & $R^{2}=0.949$ & $Q^{2}=0.681$ & \\
\hline & VIP & Full name & Block \\
\hline \multicolumn{4}{|c|}{ Staphylococcus aureus } \\
\hline CATS2D_03_LL & 3.13 & CATS2D Lipophilic-Lipophilic at lag 03 & CATS 2D \\
\hline CATS2D_04_LL & 3.13 & CATS2D Lipophilic-Lipophilic at lag 04 & CATS 2D \\
\hline H-046 & 2.92 & $\mathrm{H}$ attached to $\mathrm{C} 0(\mathrm{sp} 3)$ no $\times$ attached to next & Atom-centred fragments \\
\hline CATS2D_02_LL & 2.92 & CATS2D Lipophilic-Lipophilic at lag 02 & CATS 2D \\
\hline ALOGP & 2.92 & Ghose-Crippen octanol-water partition coeff. $(\log P)$ & Molecular properties \\
\hline CATS2D_05_LL & 2.91 & CATS2D Lipophilic-Lipophilic at lag 05 & CATS 2D \\
\hline ALOGP2 & 2.89 & squared Ghose-Crippen octanol-water partition coeff. $\left(\log P^{\wedge} 2\right)$ & Molecular properties \\
\hline $\mathrm{SssCH} 2$ & 2.85 & Sum of ssCH2 E-states & Atom-type E-state indices \\
\hline $\mathrm{SsCH} 3$ & 2.79 & Sum of ssCH3 E-states & Atom-type E-state indices \\
\hline $\log _{k}$ & 2.72 & HPLC retention factor & Experimental \\
\hline
\end{tabular}


Table 1 (continued)

\begin{tabular}{|c|c|c|c|}
\hline Descriptor & $\begin{array}{l}R^{2}=0.949 \\
\text { VIP }\end{array}$ & \multicolumn{2}{|l|}{$Q^{2}=0.681$} \\
\hline CATS2D_01_LL & 2.49 & CATS2D Lipophilic-Lipophilic at lag 01 & CATS 2D \\
\hline CATS2D_06_LL & 2.43 & CATS2D Lipophilic-Lipophilic at lag 06 & CATS 2D \\
\hline $\mathrm{C}-002$ & 2.06 & $\mathrm{CH} 2 \mathrm{R} 2$ & Atom-centred fragments \\
\hline CATS2D_00_LL & 2.06 & CATS2D Lipophilic-Lipophilic at lag 00 & CATS 2D \\
\hline BLTF96 & 2.04 & Verhaar Fish base-line toxicity from MLOGP (mmol/L) & Molecular properties \\
\hline \multirow[t]{2}{*}{ Descriptor } & $R^{2}=0.948$ & $Q^{2}=0.898$ & \\
\hline & VIP & Full name & Block \\
\hline \multicolumn{4}{|c|}{ Staphylococcus epidermidis } \\
\hline CATS2D_03_LL & 2.83 & CATS2D Lipophilic-Lipophilic at lag 03 & CATS 2D \\
\hline CATS2D_04_LL & 2.83 & CATS2D Lipophilic-Lipophilic at lag 04 & CATS 2D \\
\hline H-046 & 2.79 & $\mathrm{H}$ attached to $\mathrm{C} 0(\mathrm{sp} 3)$ no $\times$ attached to next & Atom-centred fragments \\
\hline CATS2D_02_LL & 2.79 & CATS2D Lipophilic-Lipophilic at lag 02 & CATS 2D \\
\hline $\mathrm{SssCH} 2$ & 2.78 & Sum of ssCH2 E-states & Atom-type E-state indices \\
\hline CATS2D_05_LL & 2.77 & CATS2D Lipophilic-Lipophilic at lag 05 & CATS 2D \\
\hline $\mathrm{SsCH} 3$ & 2.66 & Sum of ssCH3 E-states & Atom-type E-state indices \\
\hline ALOGP & 2.64 & Ghose-Crippen octanol-water partition coeff. $(\log P)$ & Molecular properties \\
\hline ALOGP2 & 2.56 & squared Ghose-Crippen octanol-water partition coeff. $\left(\log P^{\wedge} 2\right)$ & Molecular properties \\
\hline CATS2D_01_LL & 2.51 & CATS2D Lipophilic-Lipophilic at lag 01 & CATS 2D \\
\hline CATS2D_06_LL & 2.44 & CATS2D Lipophilic-Lipophilic at lag 06 & CATS 2D \\
\hline $\log _{k}$ & 2.24 & HPLC retention factor & Experimental \\
\hline C-002 & 2.18 & $\mathrm{CH} 2 \mathrm{R} 2$ & Atom-centred fragments \\
\hline CATS2D_00_LL & 2.18 & CATS2D Lipophilic-Lipophilic at lag 00 & CATS 2D \\
\hline CATS2D_07_LL & 1.98 & CATS2D Lipophilic-Lipophilic at lag 07 & CATS 2D \\
\hline $1+2+0$ & $R^{2}=0.949$ & $Q^{2}=0.841$ & \\
\hline Descriptor & VIP & Full name & Block \\
\hline \multicolumn{4}{|l|}{ QSTR } \\
\hline CATS2D_03_LL & 3.12 & CATS2D Lipophilic-Lipophilic at lag 00 & CATS 2D \\
\hline CATS2D_04_LL & 3.12 & CATS2D Lipophilic-Lipophilic at lag 04 & CATS 2D \\
\hline ALOGP2 & 3.00 & Squared Ghose-Crippen octanol-water partition coeff. $\left(\log P^{\wedge} 2\right)$ & Molecular properties \\
\hline ALOGP & 2.94 & Ghose-Crippen octanol-water partition coeff. $(\log P)$ & Molecular properties \\
\hline $\mathrm{H}-046$ & 2.88 & $\mathrm{H}$ attached to $\mathrm{C} 0(\mathrm{sp} 3)$ no $\times$ attached to next & Atom-centred fragments \\
\hline CATS2D_02_LL & 2.88 & CATS2D Lipophilic-Lipophilic at lag 02 & CATS 2D \\
\hline CATS2D_05_LL & 2.86 & CATS2D Lipophilic-Lipophilic at lag 05 & CATS 2D \\
\hline $\mathrm{SssCH} 2$ & 2.82 & Sum of ssCH2 E-states & Atom-type E-state indices \\
\hline $\mathrm{SsCH} 3$ & 2.79 & Sum of ssCH3 E-states & Atom-type E-state indices \\
\hline $\log _{k}$ & 2.71 & HPLC retention factor & \\
\hline CATS2D_01_LL & 2.43 & CATS2D Lipophilic-Lipophilic at lag 01 & CATS 2D \\
\hline CATS2D_06_LL & 2.35 & CATS2D Lipophilic-Lipophilic at lag 06 & CATS 2D \\
\hline BLTF96 & 2.14 & Verhaar Fish base-line toxicity from MLOGP (mmol/L) & Molecular properties \\
\hline BLTD48 & 2.14 & Verhaar Daphnia base-line toxicity from MLOGP (mmol/L) & Molecular properties \\
\hline MLOGP & 2.14 & Moriguchi octanol-water partition coeff. $(\log P)$ & Molecular properties \\
\hline
\end{tabular}

$R^{2}$ denotes coefficient of determination for the model, $Q^{2}$ denotes cross-validated coefficient of determination for the model 
the negatively charged phospholipids, seems to be the major factor of interaction with lipopeptides. In case of S. epidermidis, B. subtilis concentration of PG is similar (67 vs 70\%), and the observed MIC values are the lowest. On the other hand, the E. faecalis membrane contains only $20 \%$ PG and the MIC values are the highest. Whereas in case of $S$. aureus the percent of PG in the membrane is $40 \%$, so it is moderate among tested microbes, and also the moderate activity of lipopeptides were noticed (Fig. 1).

When we look inside of the obtained QSAR models, additional conclusions can be drawn. The most important descriptors used for building of OPLS models are the same in their nature. They are related to lipophilicity properties, such as CATS descriptors, Ghose-Crippen octanol-water partition coefficient (ALOGP) as well as the number of $\mathrm{C}$ atoms. The special attraction of CATS descriptors is its exhaustive 2D pharmacophore/biophore model based on the cross-correlation of generalized atom types (Schneider et al. 1999). Its usefulness for QSAR studies indicated several reports (Ahmed et al. 2013; Reutlinger et al. 2013). Furthermore, the chromatographically obtained parameters $\left(\log _{k}\right)$, which can be interpreted as chromatographic lipophilicity index, have a similar impact like calculated lipophilicity.
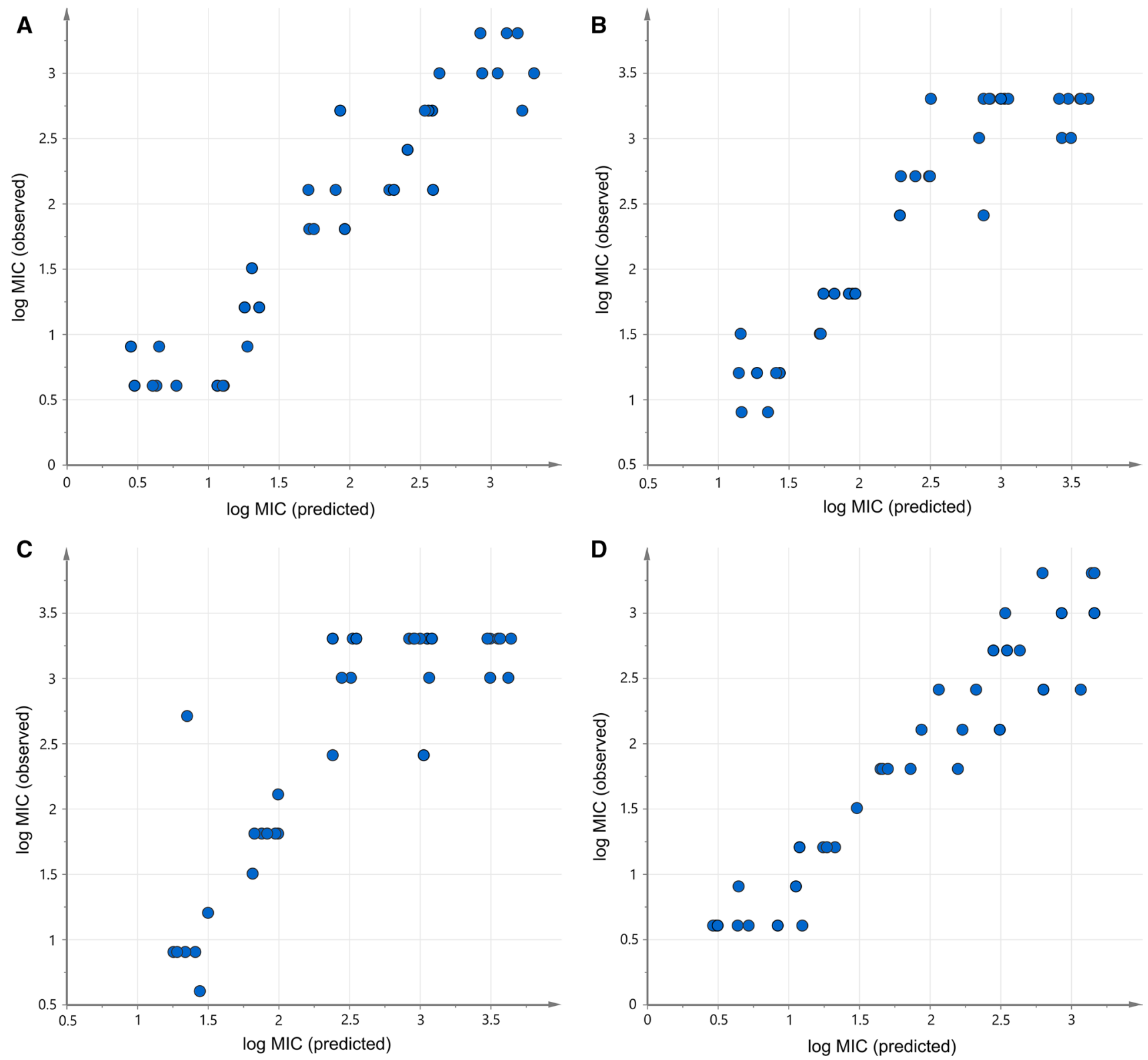

Fig. 1 The comparison of performance for the obtained QSAR models for each strain of bacteria: a Bacillus subtilis, b Staphylococcus aureus, c Staphylococcus epidermidis and $\mathbf{d}$ Enterococcus faecalis 
This finding highly indicated that $\log _{k}$ reflects lipophilic properties of lipopeptides and can be concerned as $\log P$ surrogate. The traditional scales of lipophilicity is based on partition coefficient between two phases, $n$-octanol and water, a system that is conventionally used due to its partitioning analogy with the biological environment. However, the traditional approach (so-called shake flask method) has significant limitations. It is laborious, time-consuming, requires pure substances in large quantities. Moreover, the compounds which exhibit surface-active properties, as investigated lipopeptides, cannot be analyzed in this way. Therefore, the chromatographic approach was used to assess lipophilicity of this class of chemical derivatives. To gain more insight into molecular mechanism of retention, the QSRR approach was used. The lipophilicity index measured by HPLC is derived by the retention time that is converted to the logarithm of the retention factor $\log _{k}$ (Dreher et al. 2017). The "one-run gradient method" was describes in the literature as an attractive alternative to performing several isocratic runs followed by extrapolation (Giaginis and Tsantili-Kakoulidou 2008; Liang et al. 2017).

As a means to investigate the relationship between molecular properties and retention, firstly the MLR regression was applied. The best MLR model that includes three descriptors (sum of sCH3 E-states [SsCH3], sum of sNH2 E-states [SsNH2] and frequency of $\mathrm{C}-\mathrm{C}$ at topological distance $9[\mathrm{~F} 09[\mathrm{C}-\mathrm{C}]])$, is presented below:

\begin{tabular}{l}
$\log _{k}=-2.544( \pm 0.492)+1.589( \pm 0.212) \mathrm{SsCH}_{3}$ \\
$\frac{-0.029( \pm 0.005) \mathrm{SsNH}_{2}+0.012( \pm 0.002) \mathrm{F} 09[\mathrm{C}-\mathrm{C}]}{1.32 \times 10^{-5}} \quad 1.97 \times 10^{-8} 1.36 \times 10^{-5} 2.10 \times 10^{-4}$ \\
\hline$R=0.955 \quad R^{2}=0.913 \quad F=107.656 \quad s=0.034 \quad p=1.96 \times 10^{-14}$
\end{tabular}

As might be expected the increased number of $\mathrm{C}$ atoms in carbon chain leads to increased retention. Oppositely, sum of $\mathrm{sNH} 2 \mathrm{E}$-states, a group that influences the polarity of the molecule, reduces retention of investigated lipopeptides. The $\mathrm{NH}_{2}$ group can be responsible for interaction with polar mobile phase. The result of PLS and OPLS regression analysis are presented in Table 3S. The statistical parameters of all obtained QSRR models are similar. The most important factors, according to VIP value are listed in Table 3S. The descriptors that highly influence the value of chromatographic parameter $\log _{k}$ are connected with calculated lipophilicity (ALOGP and MLOGP descriptors but also CATS descriptors) and the number of $\mathrm{C}$ atoms in a molecule. Oppositely to MLR model, the influence of $\mathrm{NH}_{2}$ group was not underlined in the obtained PLS and OPLS models. However, the calculated lipophilicity indexes can include this information, since the MLOG and ALOGP calculation algorithms use the whole structure and all functional groups of a molecule. Summarizing the QSRR analysis, the $\log _{k}$ parameter reflects very well with lipophilic properties of investigated lipopeptides.

The last step of our study concerned QSTR. One of the factors limiting the clinical use of lipopeptides is their hemolytic characters. Although, the coarse-grained molecular dynamics simulations revealed no association between the lipopeptides and model mammalian bilayers, the hemolytic properties of lipopeptides were previously reported (Greber et al. 2017). It should be noticed that the hemolytic concentration of lipopeptides is significantly higher as antimicrobial, but it still limits clinical use of AMPs. The obtained QSTR models (Table 1) suggested that lipopeptides degrade cell membranes of erythrocytes in the same way as bacterial membranes. Descriptors obtained in the QSTR-OPLS model are very similar to those previously described in QSAR models, belong to the same class and they are connected with lipophilic properties of target compounds.

\section{Conclusion}

The obtained results suggested that the simple HPLC method could be used for lipophilicity assessment of short cationic lipopeptides. Furthermore, the chromatographic indexes can be useful for prediction of antibacterial activity. Summarizing, the QSAR and QSTR analysis, all obtained models indicate that lipophilicity play vital role. This result is not surprising since lipophilicity is well known as the physicochemical parameter that determines biological properties of xenobiotics. The most important conclusion is the fact that lipopeptides show a nonspecific interaction between erythrocytes and bacterial membranes. Different affinities between mammalian and bacterial bilayers seem to be the vital point to design more active and less toxic antimicrobial lipopeptides.

\section{Compliance with ethical standards}

Conflict of interest Katarzyna E. Greber, Krzesimir Ciura, Mariusz Belka, Piotr Kawczak, Joanna Nowakowska, Tomasz Bacczek and Wiesław Sawicki confirm that this article content has no conflicts of interest.

Ethical approval The article does not contain any studies in patients by any of the authors.

Open Access This article is distributed under the terms of the Creative Commons Attribution 4.0 International License (http://creativecommons.org/licenses/by/4.0/), which permits unrestricted use, distribution, and reproduction in any medium, provided you give appropriate credit to the original author(s) and the source, provide a link to the Creative Commons license, and indicate if changes were made. 


\section{References}

Ahmed L, Rasulev B, Turabekova M, Leszczynska D, Leszczynski J (2013) Receptor- and ligand-based study of fullerene analogues: comprehensive computational approach including quantumchemical, QSAR and molecular docking simulations. Org Biomol Chem 11:5798-5808. https://doi.org/10.1039/c3ob40878g

Alexander DLJ, Tropsha A, Winkler DA (2015) Beware of R2: simple, unambiguous assessment of the prediction accuracy of QSAR and QSPR models. J Chem Inf Model 55:1316-1322

Bechinger B, Lohner K (2006) Detergent-like actions of linear amphipathic cationic antimicrobial peptides. Biochim Biophys Acta 1758:1529-1539

Brogden KA (2005) Antimicrobial peptides: pore formers or metabolic inhibitors in bacteria? Nat Rev Micro 3:238-250

User Guide to SIMCA (2017) By MKS Umetrics Version 13. http:// chemsrv0.pph.univie.ac.at/scripten/EDV/Software/Simca13/ User\%20Guide\%20to\%20SIMCA\%2013.pdf. Accessed 6 Nov 2017

Ciura K, Nowakowska J, Rudnicka-Litka K, Kawczak P, Bączek T, Markuszewski MJ (2016) The study of salting-out thin-layer chromatography and their application on QSRR/QSAR of some macrolide antibiotics. Monatshefte fur Chemie 147:301-310. https:// doi.org/10.1007/s00706-015-1606-5

Clinical and Laboratory Standards Institute (2012) Methods for dilution antimicrobial susceptibility tests for bacteria that grow aerobically; approved standard-9th edition. CLSI document M07A9, vol 32, issue 2. Clinical and Laboratory Standards Institute, Wayne, PA

Clinical and Laboratory Standards Institute (2017) Performance standards for antimicrobial susceptibility testing, 27th edition. CLSI supplement M100, Wayne, PA

Colomb-Cotinat M, Lacoste J, Brun-Buisson C, Jarlier V, Coignard B, Vaux S (2016) Estimating the morbidity and mortality associated with infections due to multidrug-resistant bacteria (MDRB), France, 2012. Antimicrob Resist Infect Control 12(5):56. https:// doi.org/10.1186/s13756-016-0154-z

Dragon 7 molecular descriptors (2017) https://chm.kode-solutions.net/ products_dragon.php. Accessed 06 Nov 2017

Dreher J, Scheiber J, Stiefl N, Baumann K (2017) xMaP - An interpretable alignment-free $4 \mathrm{D}-\mathrm{QSAR}$ technique based on molecular surface properties and conformer ensembles. J Chem Inf Model. https://doi.org/10.1021/acs.jcim.7b00419 (Epub ahead of print)

Giaginis C, Tsantili-Kakoulidou A (2008) Current state of the art in HPLC methodology for lipophilicity assessment of basic drugs. a review. J Liq Chromatogr Relat Technol 31:79-96

Greber KE, Dawgul M (2017) Antimicrobial peptides under clinical trials. Curr Top Med Chem 17:620-628
Greber KE, Dawgul M, Kamysz W, Sawicki W (2017) Cationic net charge and counter ion type as antimicrobial activity determinant factors of short lipopeptides. Front Microbiol 8:123. https://doi. org/10.3389/fmicb.2017.00123

Horn JN, Sengillo JD, Lin D, Romo TD, Grossfield A (2012) Characterization of a potent antimicrobial lipopeptide via coarse-grained molecular dynamics. Biochim Biophys Acta 1818:212-218. https://doi.org/10.1016/j.bbamem.2011.07.025

HyperChem Computational Chemistry (1996) Part 1 practical guide. Part 2 theory and methods. Hypercube Inc., Waterloo

Liang C, Qiao JQ, Lian HZ (2017) Determination of reversed-phase high performance liquid chromatography based octanol-water partition coefficients for neutral and ionizable compounds: methodology evaluation. J Chromatogr A 15(1528):25-34. https://doi. org/10.1016/j.chroma.2017.10.064

odeschini RT, onsonni VC (eds) (2009) Molecular descriptors for chemoinformatics: Volume I: alphabetical listing/volume II: appendices, references, vol 1. Wiley-VCH Verlag GmbH \& CoKGaA, Weinheim. https://doi.org/10.1002/9783527628766.ch22

Reutlinger M, Koch CP, Reker D, Todoroff N, Schneider P, Rodrigues T, Schneider G (2013) Chemically advanced template search (CATS) for Scaffold-hopping and prospective target prediction for "Orphan" molecules. Mol Inform 32:133-138

Roy K, Kar S, Das RN (2015) A primer on QSAR/QSPR modeling, fundamental concepts. Springer International Publishing, Switzerland. https://doi.org/10.1007/978-3-319-17281-1

Saxena AK, Prathipati P (2003) Comparison of MLR, PLS and GAMLR in QSAR analysis. SAR QSAR Environ Res 14:433-445

Schneider G, Neidhart W, Giller T, Schmid G (1999) "Scaffold-hopping" by topological pharmacophore search: a contribution to virtual screening. Angew Chem Int Ed Engl 38:2894-2896

Shai Y, Oren Z (2001) From "carpet" mechanism to de-novo designed diastereomeric cell-selective antimicrobial peptides. Peptides 22:1629-1641

Tropsha A, Gramatica P, Gombar VK (2003) The Importance of being earnest: validation is the absolute essential for successful application and interpretation of QSPR models. QSAR Comb Sci 22:69-77

Worley B, Powers R (2013) Multivariate analysis in metabolomics. Curr Metab 1:92-107 